\title{
Civilisations
}

Revue internationale d'anthropologie et de sciences

humaines

42-2 | 1993

enQuete d'identité

\section{Identité régionale et fêtes contemporaines}

\author{
Andreas C. Bimmer
}

URL : http://journals.openedition.org/civilisations/2388

DOI : 10.4000/civilisations.2388

ISSN : 2032-0442

Éditeur

Institut de sociologie de l'Université Libre de Bruxelles

Édition imprimée

Date de publication : 1 décembre 1993

Pagination : 243-247

ISBN : 2-87263-108-9

ISSN : 0009-8140

\section{Référence électronique}

Andreas C. Bimmer, «Identité régionale et fêtes contemporaines », Civilisations [En ligne], 42-2 | 1993, mis en ligne le 01 décembre 1996, consulté le 04 mai 2019. URL : http://journals.openedition.org/ civilisations/2388; DOI : 10.4000/civilisations.2388

Ce document a été généré automatiquement le 4 mai 2019.

(c) Tous droits réservés 


\title{
Identité régionale et fêtes contemporaines
}

\author{
Andreas C. Bimmer
}

1 Dans une perspective patrimoniale - qui fût pendant longtemps celle de l'ethnologie en général - on a considéré les fêtes traditionnelles comme rendant compte d'une identité locale, régionale ou nationale. On peut se demander dès maintenant quelle est l'influence de ce qu'on appelle "identité » sur la fête, d'une part, et quel pourrait être le rôle de la " régionalité », de l'appartenance à un espace géographique déterminé, d'autre part. La question plus importante encore est la suivante : les fêtes actuelles, "traditionnelles » ou " modernes », peuvent-elles contribuer à la formation d'une identité régionale ou non? C'est en résumé le propos de mon intention.

2 La recherche sur la fête a une longue tradition dans l'ethnologie allemande. Son approche a sensiblement changé dans les années, quand la Deutche Volkskunde commença à se reconsidérer comme science sociale pour devenir une Europäiche Ethnologie. Dès lors, la fête comme phénomène, comme fait isolé, a cessé d'être le seul but de la recherche pour faire place aux fonctions et structures sociales intégrant la fête dans leur unité. On s'interroge maintenant plutôt sur les éléments constitutifs d'une fête et de son impact sur le groupe exerçant cette manifestation. On ne parle plus de «Sitte und Brauch " (coutume et usage) ou de "Fest und Feier» (fête et spectacle) dans la tradition de la Volkskunde mais seulement de "Brauch» comme processus social qui change sa signification, son déroulement et sa fonction sociale en fonction des intérêts du groupe qui l'exerce, de son milieu socio-économique et de la situation historique. La description phénoménologique pure ne fait plus recette.

3 Les recherches sur la fête, menées dans les dix dernières années en Allemagne, portent principalement, à mon avis, sur les grands points suivants :

1. L'interprétation générale aussi bien qu'analytique des fêtes sous l'angle théorique et social.

2. L'analyse détaillée des fêtes particulières d'un point de vue historique et ethnologique.

3. Des fêtes sous l'angle géographique spécifique à chaque région. 
4. Des problèmes supplémentaires apparus à la suite des recherches théoriques et monographiques.

Il $\mathrm{y}$ a principalement quatre institutions en Allemagne où on travaille de manière intensive et depuis longtemps sur la fête.

Commençons avec les collègues de Munich d'où nous viennent des études détaillées sur la distribution géographique des fêtes. Sous la direction de Helge Gerndt, le projet « So feiern die Bayern "(Les Bavarois et leurs manières à fêter) a essayé d'établir un inventaire complet des fêtes actuelles en Bavière. Une enquête a fourni le cadre de l'analyse et les résultats en ont été présenté lors d'une grande exposition.

Il faut mentionner ensuite les travaux menés à Mayence à l'initiative de Herbert Schwedt concernant la Fastnacht. A l'origine il a étudié les coutumes de la Fastnacht de Tübingen au sud-ouest de l'Allemagne. Lui et ses collaborateurs ont traité les phénomènes les plus spectaculaires de cette fête, organisée dans une ville (celle de Mayence). Tout au contraire de Gerndt il n'a pas mis au premier plan une région ou une province avec toutes les facettes de petites fêtes de campagne mais il s'est occupé d'une seule fête urbaine avec tous les aspects de son organisation. Les travaux ont inclus par exemple - et c'était unique à ce moment - des non-visiteurs: il s'agit des habitants de Mayence qui ne voulaient pas fêter la Fastnacht et qui n'avaient que le choix de sortir de la ville.

Il y a quelques années, Herbert Schwedt a publié des études très importantes sur un pays fédéral (Rheinland-Pfalz) où on aime faire la fête comme en Bavière (Schwedt et Schwedt, 1989). Ces enquêtes concernent de nouvelles fêtes essayant d'identifier les raisons de leur naissance. Le résultat est intéressant mais pas étonnant: dans la plupart des cas, les motifs relevaient du domaine de l'économie et du tourisme.

La plupart de ces fêtes n'ont pas de tradition. Parfois elles se basent sur une kermesse - et on peut dire qu'il y a là un nouveau type de fête villageoise. Il est modelé par trois catégories d'intérêts : l'économie, la politique et en dernière ligne le spectacle et la joie pour les participants. De ce fait, il est important d'interpréter la fête d'arrondissement dans une perspective plus complexe, voire dans le cadre des recherches sur la ville en tant que telle.

En considérant ainsi la fête urbaine par rapport au commerce et à la politique locale et provinciale, la création identitaire par les fêtes reçoit une autre dimension. Dans ce contexte Ina Maria Greverus a posé la question provocante : "Avons-nous besoin de fêtes " (Greverus, 1977). Dans le même style provocant, le suédois Nils Arvid Bringéus - toujours à propos des fêtes urbaines - titrait dans un essai en Hessische Blatter für Volks - und Kulturforschung : «Pas de cérémonieje vous en prie »(Bringéus, 1978).

10 Il faut ajouter sur cette liste nos propres recherches menées a Marbürg sur le Hessentag (les fêtes organisées pour le " jour du pays de Hessen »). Chaque année, le Hessentag a lieu dans une ville différente de Hessen; il est organisé comme un spectacle qui mène grand train. Le Hessentag sert comme autoreprésentation du gouvernement de Hessen dans le cadre détendu d'une fête, en prétendant à l'unité de tradition et de modernité ainsi que des costumes traditionnels et des techniques. Le tout inséré dans les activités politiques du pays de Hessen. Le Hessentag est un modèle exemplaire du folklore politique et ce fut tout particulièrement cet aspect qui a retenu notre attention.

11 Les buts de Hessentag sont expressément formulés dans une loi du pays de Hessen : c'est, en premier lieu, le renforcement de la solidarité de tous les gens de Hessen. En effet, après la guerre, la population de Hessen comptait $25 \%$ de réfugiés et le problème de leur 
intégration - comme aujourd'hui celle des travailleurs étrangers - était d'une grande importance pour les dirigeants de la région.

Le programme de la fête est un mélange subtilement dosé.

13 Sous le titre: "Le moderne et le traditionnel en Hessen » on présente des costumes et danses traditionnels ainsi que des concerts de musique moderne, des discussions avec des politiciens, des expositions sur la protection de l'environnement, etc. Tout se termine avec un grand cortège qui passe à travers une foule énorme mais aussi devant la tribune d'honneur des représentants politiques de Hessen, le Président des ministres en tête. Le cortège se donne des thématiques comme par exemple: "Nous les Hessen» ou «Tradition et progrès en Hessen » ou bien encore : « Hessen - Allemagne - Europe ».

14 Une des plus récentes inventions de cette fête est le «cortège stationnaire » : ce sont maintenant les spectateurs qui se déplacent au long du soi-disant cortège qui se déploie sur une longueur de quelques cinq kilomètres! Les petites communes ont vite repris cette idée pour ses avantages pratiques évidents. C'est le triomphe de l'anti-festif même. Le succès en chiffres du Hessentag est considérable : actuellement il ne dure plus un jour, mais bien dix et on compte environ un million de gens qui l'ont visité volontairement. Mais y a t'ilaussi un succès de l'idée?

Durant nos recherches nous avons effectué des interviews avec des gens provenant des trois niveaux de la fête :

- niveau des visiteurs ;

- niveau des acteurs, i. e. un groupe traditionnel.

- niveau des organisateurs.

On y trouve rarement quelqu'un qui déclare se sentir plus Hessen après la fête qu'avant. C'était en premier lieu le spectacle - quelque chose pour chacun - qui les a attiré.

Le Hessentag est, à notre avis, une preuve parmi d'autres que la plupart des fêtes contemporaines n'arrivent pas à toucher leur but identitaire déclaré. A une exception près: les groupes d'organisateurs - des gens de la région et des amateurs pour la plupart - dont la solidarité peut se trouver renforcée du fait de cette mise en scène en commun. A part ça, les messages qui passent le mieux par le biais de ces fêtes sont plutôt les idées politiques et économiques chères au pouvoir local que celles liées à l'unité régionale ou à l'identité locale. C'est dans ce sens-là qu'une fête comme celle du Hessentag peut être mieux comprise par rapport au concept de «folklore politique » que dans le contexte de la notion traditionnelle de fête.

Pour conclure, je dirai que ce ne sont plus les orientations spatiales ou régionales qui déterminent la formation de l'identité. Quant à la fête moderne, elle ne suffit plus pour fonder une nouvelle identité. 


\section{BIBLIOGRAPHIE}

Bimmer, Andreas C. (1987) Brauchforshung. En : Rolf W. Brednich : Grundgriss des Volkskunde.

Einführung in die Forschungfelder der Europaïschen Ethnologie ; Berlin, 311-328

Bimmer, Andreas C. Ed. (1973) : Hessentag - Ein Fest des Hessen? Anspruch und Wirklichkeit eines organisierten Volkfestes. Marburg.

Bringeus, N. A., 1978, « Bitte keine Feier oder das Fest aIs Trauma », en : Aspekte der Freizeit , Hess. Bll., 7/8, 35-49.

Greverus, I.-M., 1977, « Brauchen wir Feste ? », en : Feste in Hessen, Hessichen ,Blâtter für Volks und Kulturforschung, NF 4). 1-9.

Schwedt, H. \& Schwedt, E., 1989, Briiuche zwischen Saar und Sieg , Mainz.

\section{RÉSUMÉS}

Can the actual feasts, «traditional » or « modern » contribute to form a regional identity or not? The inquiry carried out on the "Hessentag» (Day of the Hessen country) suggests a negative answer. The way the "Hessentag" takes place illutrates the concept of "political folklore " rather than the traditional notion of a feast.

INDEX

Keywords : regional identity, cultural heritage, modernity, festivals, identity construction Mots-clés : identité régionale, patrimoine, modernité, fêtes, construction identitaire

\section{AUTEUR}

\section{ANDREAS C. BIMMER}

Université de Marburg 